\title{
IOTA: recent technology and science
}

Wesley Arthur Traub, Jean-Philippe Berger, Michael K. Brewer, Nathaniel P. Carleton, Pierre Y. Kern, et al.

Wesley Arthur Traub, Jean-Philippe Berger, Michael K. Brewer, Nathaniel P. Carleton, Pierre Y. Kern, Stefan Kraus, Marc G. Lacasse, William H. McGonagle, Rafael Millan-Gabet, John D. Monnier, Ettore Pedretti, Sam Ragland, Robert K. Reich, F. Peter Schloerb, Peter A. Schuller, Kamal Souccar, Gary Wallace, "IOTA: recent technology and science," Proc. SPIE 5491, New Frontiers in Stellar Interferometry, (20 October 2004); doi: 10.1117/12.550916

Event: SPIE Astronomical Telescopes + Instrumentation, 2004, Glasgow, United Kingdom 


\title{
IOTA: Recent Technology and Science
}

\author{
Wesley A. Traub ${ }^{a}$, J.-P. Berger ${ }^{b}$, Michael Brewer ${ }^{c}$, Nathaniel P. Carleton ${ }^{a}$, \\ Pierre $\mathrm{Kern}^{b}$, Stefan Kraus ${ }^{c, d}$, Marc G. Lacasse ${ }^{a}$, William H. McGonagle ${ }^{e}$, \\ Rafael Millan-Gabet ${ }^{f}$, John D. Monnier ${ }^{g}$, Ettore Pedretti ${ }^{a, g \dagger}{ }^{\prime}$, Sam Ragland ${ }^{a \dagger}$, \\ Robert K. Reich ${ }^{e}$, F. Peter Schloerb ${ }^{c}$, Peter Schuller ${ }^{a}$, Kamal Souccar ${ }^{c}$, and Gary Wallace ${ }^{c}$ \\ ${ }^{a}$ Smithsonian Astrophysical Observatory, 60 Garden St., Cambridge, MA 02138, USA \\ ${ }^{b}$ Laboratoire d'Astrophysique de Grenoble, BP 53 X, Grenoble Cedex 9, France \\ ${ }^{c}$ University of Massachusetts, Amherst, Dept. of Astronomy, Amherst, MA 01003, USA \\ ${ }^{d}$ Max-Planck-Institute for Radio Astronomy, Auf dem Hügel 69, 53121 Bonn, Germany \\ ${ }^{e}$ Advanced Imaging Technology Group, MIT Lincoln Laboratory, Lexington, MA 02420, USA

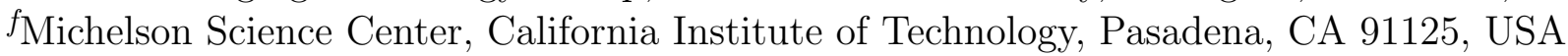 \\ ${ }^{g}$ University of Michigan Astronomy Dept., 500 Church St., Ann Arbor, MI 48109, USA
}

\begin{abstract}
Closure-phase science and technology are dominant features of the recent activity at IOTA.

Our science projects include imaging several spectroscopic binary stars, imaging YSOs including Herbig AeBe stars, detecting asymmetries in a large sample of Mira stars, and measuring water shells around Miras.

Many technology projects were pursued in order to make these science observations possible. These include installation of a third-generation integrated-optics 3-beam combiner (IONIC), completion of the real-time control system software, installation of fringe-packet tracking software, use of narrow sub- $\mathrm{H}$ band filters, validation of the phase-closure operation, development of CPLD control of the science camera (PICNIC) and star-tracker camera (LLiST), installation of a new star-tracker camera, expansion of the observing facility, and installation of new semi-automated optical alignment tools.
\end{abstract}

Keywords: infrared, interferometry, binary stars, Mira stars, software, integrated optics, closure phase

\section{INTRODUCTION}

This paper reviews progress in science and technology at the Infrared Optical Telescope Array (IOTA), since the last such review, ${ }^{1}$ some of which is reported in the present volume. ${ }^{2,3,4,5,6}$

Briefly, IOTA is a long-baseline optical interferometer located at the Smithsonian Institution's Whipple Observatory on Mount Hopkins, AZ. It comprises three 45- $\mathrm{cm}$ diameter telescopes which can be positioned at 17 stations on an L-shaped track, where the arm lengths are $15 \mathrm{~m}$ toward the south-east and $35 \mathrm{~m}$ toward the north-east. IOTA operated with 2 telescopes from 1995-2003, and with 3 telescopes since February 2002. The interferometer ${ }^{7,1}$ has been used as a testbed for new cutting-edge technologies, $, 8,9,10$ and has produced recent science results in the 2 and 3 -telescope configuration. ${ }^{11,12,13,14,15,16,17,18,19,20}$

In a nutshell, the three beams arriving from the vacuum delay-line tank each hit their own individual dichroic mirror which separates the visible and infrared light. Each visible beam continues toward the star tracker servo system. Each science infrared beam is reflected toward a flat mirror and then to an off-axis parabola which focuses it onto a single-mode, polarization-maintaining $\mathrm{H}$-band fiber, and thence to the IONIC-3T integratedoptics beam-combiner. ${ }^{21}$

The balance of this paper focuses on specific technical and scientific advances at IOTA.

${ }^{\dagger}$ Michelson Postdoctoral Fellow

Further author information: Send correspondence to W.A.T., email: wtraub@cfa.harvard.edu 


\section{BINARY STAR IMAGES}

Three binary systems have been imaged at IOTA, in H-band, using our new closure-phase capability. One of these is Capella A, shown in Fig. 1. The inset is the beam pattern, and the contours are $5 \%$ intervals. The coordinates are centered on Capella Aa, the brighter infrared component, with a diameter of 8.4 mas. Its slightly fainter companion is Capella $\mathrm{Ab}$, at a separation of about 44 mas, has diameter 6.3 mas. During the 5 consecutive days of observation the angle between the stars changed by about $14^{\circ}$ clockwise, which motion was removed in order to make this image. Only two telescope configurations were used to make this map. The other binaries imaged at IOTA are $\lambda$ Vir, and the Wolf-Rayet star WR 140, a binary that had previously been imaged by aperture masking at the Keck telescope.

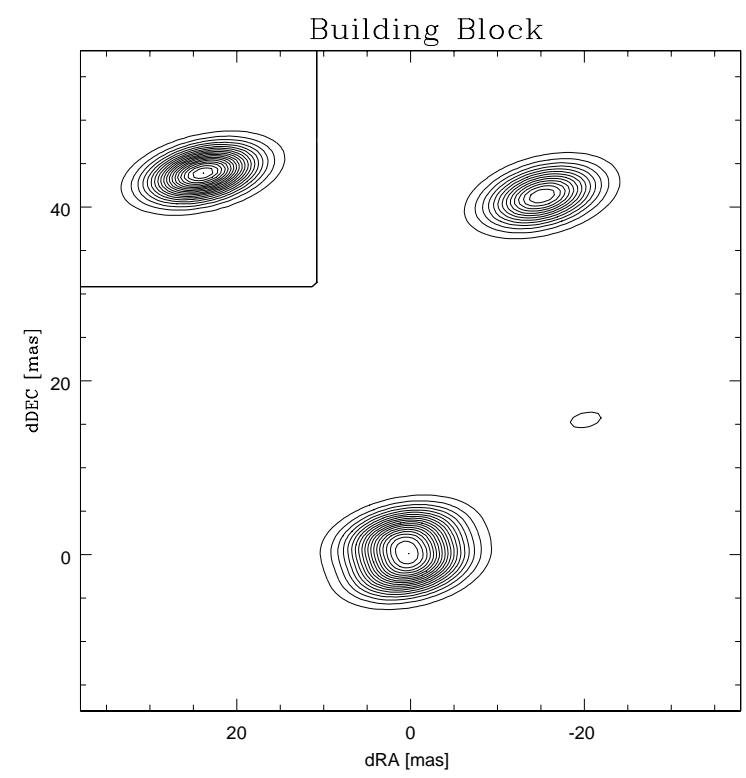

Figure 1. Aperture synthesis map of Capella on 12-16 Nov. 2002, using one of three different reconstruction algorithms, all of which performed approximately equally well. ${ }^{3}$

\section{AGB-STAR ASYMMETRIES}

We have used the 3-telescope IOTA to measure the closure phase on 56 AGB stars, most of which are Miras, plus some semi-regular variables ${ }^{22}$ We find that about $25 \%$ of these stars show significant non-zero closure phases, ranging from about $\pm 4^{\circ}$ to $\pm 160^{\circ}$, as shown in Fig. 2. The asymmetry that we measure is that part of the brightness distribution which cannot be made symmetric with respect to a reflection through a point. We speculate that these asymmetries may be caused by circumstellar dust or gas, or by flux from an as-yet undetected companion. Based on the results of this survey, we intend to focus future observations on these asymmetric AGB stars, using the imaging power of three interferometers, IOTA, VLBA, and ISI, all of which have phase-closure capabilities, and each of which observes in a different wavelength regime. Thus with VLBA we can observe $\mathrm{SiO}$ masers in a shell around these stars, a project which has already produced results. ${ }^{23}$ And with the U.C. Berkeley Infrared Spatial Interferometer (ISI) ${ }^{24}$ the dust shell around these stars can be observed. The dust shell appears to lie just outside the $\mathrm{SiO}$ maser shell. Collectively, using all three observatories, our goal will be to search for correlations between asymmetries in these three shells, and thereby shed light on the mass loss process around AGB stars. 


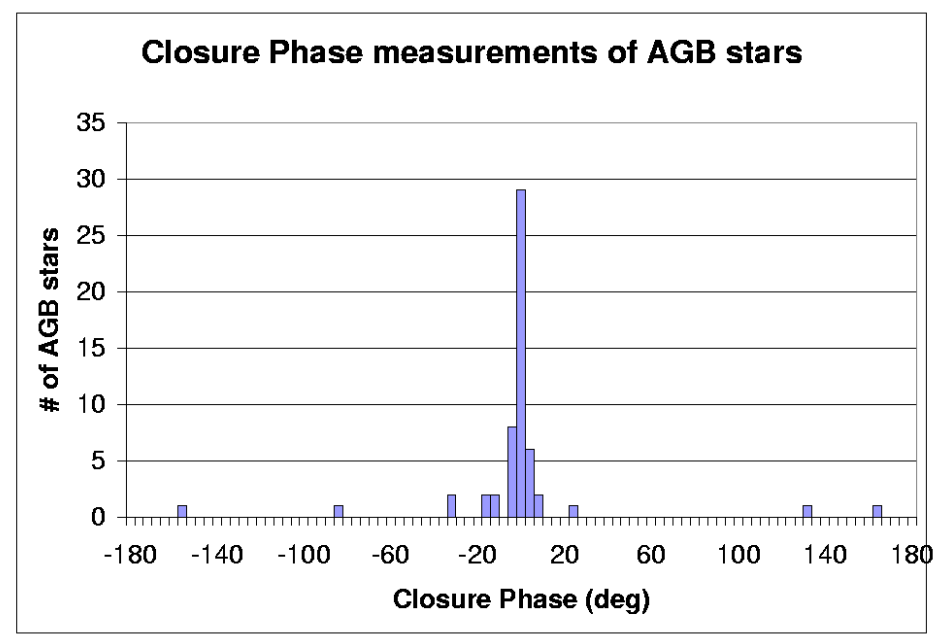

Figure 2. Closure phase measurements of 56 AGB stars observed in 2002 and 2003 with the IOTA 3-telescope interferometer. Each measurement represents at least 5 minutes of on-target integration.

\section{MIRA WATER SHELLS}

In a program designed to probe the mystery of why Mira-type stars appear to have different diameters depending on the wavelength of observation, Perrin and others started a program of narrow-band filter observations at IOTA using the FLUOR two-beam K-band combiner, in the years 2000-2002. The results are reported in a recent paper, ${ }^{25}$ where it is shown that the optical depth toward many Miras is greater at the edges of the $\mathrm{K}$ band than at the center. This result can be interpreted in terms of a shell of hot water molecules around each star, plus some $\mathrm{CO}$ which further enhances the depth on the long-wavelength edge of the $\mathrm{K}$ band. This result is qualitatively in agreement with studies of Mira spectra, particularly in the infrared and from space.

Building on these data, we have calculated the spectrum of hot $\mathrm{H}_{2} \mathrm{O}$ and $\mathrm{CO}$, and applied these results to the observed Miras (Traub et al., in preparation). We find that the opacities can be explained by a relatively large amount of water, with implications for the mass loss mechanism around these stars.

\section{INTEGRATED-OPTICS 3-BEAM COMBINER}

The current integrated-optics 3-beam combiner was installed in November 2002, and has been used continuously since then. Some characteristics of this combiner are described in this volume by Ragland et al. ${ }^{5}$

Briefly, the combiner was built in Grenoble, and designed for H-band operation. The three telescope beams are focussed onto three single-mode fibers, and these fibers are bonded to the input end of the thin glass plate that contains the combiner. Each input beam is split into 2 beams by a $\mathrm{Y}$ junction, and these are then combined pairwise in $\mathrm{X}$ junctions, producing 6 output beams. These beams emerge from the opposite end of the glass plate into air, and are focussed by a pair of lenses onto 6 corresponding pixels of our PICNIC detector.

The path differences inside the combiner between the splitter and combiner junctions are additive to the intrinsic phases of the beams, and thus to the closure phase. We find that the paths are very stable with time, and add negligibly to the closure phase uncertainty, as best we can tell. Closure phase uncertainties of about $1^{\circ}$ are typical, from a set of 500 scans, for example. See the paper by Pedretti et al. ${ }^{4}$ for details on the extraction of closure phases, and measured statistical accuracies. 
The combiner has also been used with as set of 3 narrow band filters that span the normal H-band range, ${ }^{2}$ and from these measurements we have learned about the small but real color differences of internal additive phase. There is also a small effect due to polarization, but normally this combiner is used without any polarizing optics. The raw closure phase also depends on the timing of samples, but this is not intrinsic to the combiner, but rather it is caused by the sequential nature of the sampling of our detector; the effect drops out in calibration, assuming that the same timing is used on the calibrator star.

\section{CONTROL SYSTEM SOFTWARE}

The IOTA Interferometer Control System (ICS) has been developed using the hardware and software architecture of the Large Millimeter Telescope Monitor and Control System (LMTMC). This system provides an efficient framework for monitoring, control, and data acquisition in a distributed computing environment. An overview cartoon is shown in Fig. 3.

When using the LMTMC framework, telescope system components are described in XML configuration files, and source code for extendable base classes and user interfaces are automatically generated by the LMTMC system. These implement a global state system and provide underlying communication methods, insuring consistency and allowing flexibilty.

The ICS is a set of programs, created within this LMTMC framework, which run together in a coordinated way. These may be summarized in two major categories: (1) the real time control programs; and (2) the programs which provide the interface between the user and the real time programs. The real time programs run on VME-based Motorola PowerPC single board computers running VxWorks. The interface programs run on a Sun workstation running Solaris.

The user interface includes tools that allow the user to compose observing programs, perform monitor and control functions, execute database logging and retrieval, and to plot observing and engineering data in a variety of ways.

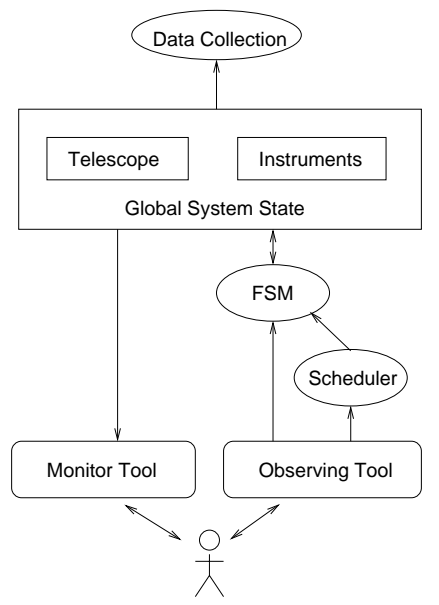

Figure 3. Conceptual diagram of the IOTA control system. FSM is finite-state machine.

\section{CPLD CAMERA CONTROLLERS}

We use CPLD controllers to generate the readout timing signals for our star-tracker CCD and also our PICNIC science camera. For the recently-updated star-tracker, the code has been rewritten to handle the new output format, and various readout modes (full frame, and several binned modes). For the science camera, the readout code was rewritten to accomodate not only our previous 6-spot mode, but also the new 6-dispersed-spot mode. 


\section{FRINGE-PACKET TRACKING}

Real-time fringe tracking and control is crucial to the success of phase-closure measurements. The reason for this is that the fringes from each telescope pair must be measured at nearly the same time, so that we are sampling the same set of path delays through the atmosphere between each pair. It probably is allowable to have the fringe packets controlled to be within a few wavelengths of being simultaneous, but the measurements should certainly be made on each fringe packet at the same time, coordinated to within a small fraction of an atmospheric coherence time. The measurement cadence can be made faster or slower, but the longer this time becomes, the more likely one is to see abrupt atmospheric phase changes occur in the path of at least one of the beams.

We have developed and compared 3 different fringe-tracking algorithms in simulations as well as in real-time tracking at IOTA. ${ }^{4,2}$ We find that all three perform about equally well on bright stars, but on faint stars two of the algorithms emerge as superior at this point. The test metric was to run each algorithm in real time at IOTA, observing a real star, and compare the resulting mean value of closure phase for a 500 -scan sequence. On a bright calibrator star $(\mathrm{H}=4.9)$ the three algorithms yielded closure phases that were identical to within a maximum spread of $0.6^{\circ}$, with errors of about $0.8^{\circ}$ on average. On a fainter target star $(\mathrm{H}=6.6)$ the spread in closure phases was $12^{\circ}$, with uncertainties of about $2.5^{\circ}$ on two of the methods (and mean values in agreement with those uncertainties), but an uncertainty of $6.9^{\circ}$ on the third method, and a comparably shifted mean value as well. Thus the mean values and uncertainties seem to be consistent. The third method is currently being revised in light of this experience, and further tests are planned to continue this process of optimization.

\section{NARROW FILTERS AND PRISM DISPERSER}

We are currently equipped with narrow-band filters in $\mathrm{H}$ and $\mathrm{K}$ bands, as well as new direct-view dispersive prisms that cover JHK. At both $\mathrm{H}$ and $\mathrm{K}$ we have cooled interference filters that can be rotated into the beam just in front of the PICNIC detector. Both sets of filters are narrow in the sense that they are about $1 / 3$ the width of a conventional wide-band $\mathrm{H}$, or about $1 / 5$ the width of the $\mathrm{K}$ filter, and they are roughly contiguous in coverage, so that together they sample the blue wing, center, and red wing of the bandpass. This arrangement gives us a modest wavelength leverage on targets that are nominally independent of wavelength, but more important it gives us coverage in the continuum and absorption wings of targets that have $\mathrm{H}_{2} \mathrm{O}$ or $\mathrm{CO}$ in their spectra, e.g., AGB and Miras in particular.

We recently designed and fabricated two types of direct-view prisms that can be used simultaneously on all of $\mathrm{J}, \mathrm{H}$, and $\mathrm{K}$ bands if desired, or on just one of these bands. The prisms are in front of the detector dewar, at room temperature, and therefore more useful in $\mathrm{J}$ and $\mathrm{H}$ than in $\mathrm{K}$ (where the thermal background would add significantly to each dispersed spectral element). The prisms are made of calcium fluoride and water-free quartz. The "small" prism disperses $\mathrm{H}$ into about 3 spectral elements, and the "large" prism disperses $\mathrm{H}$ into about 7 elements. Roughly similar dispersions hold for $\mathrm{J}$ and K. The spectra will be dispersed across the PICNIC detector pixels so that all 6 output channels of the integrated optics combiner can be viewed simultaneously. We are currently testing the CPLD readout code to implement this mode of observation.

\section{PHASE-CLOSURE VALIDATION}

We have measured the response of our 3-beam integrated-optics combiner as a function of target color, stability over time, polarization, and detector readout timing. A preliminary report is in this volume. ${ }^{5}$

On color, with a wide-band $\mathrm{H}$ filter, we find that the closure phase is independent of the color temperature of the target star, within measurement error. With the narrow-band filters, we find that the closure phase varies slightly, if at all, with filter choice, with the exception that the longest-wavelength narrow filter shows about a $10^{\circ}$ difference in closure phase when measured with 3 detector outputs compared to the corresponding 3 complementary outputs.

On stability, we find that over many nights, and using a variety of calibrator stars, the closure phase stays within a peak-peak range of at most $5^{\circ}$. However in actual operation, on a given night, and on the same 
calibrator star, the rms variation of closure phase is less than about $1^{\circ}$, and may be substantially less, being limited more by photon statistics than any property of the combiner itself.

On polarization, we normally make all our observations with this combiner in unpolarized light. However as a test, when we insert a linear infrared polarizer into the output beams, we find that the closure phase changes by $1^{\circ}$ or less in two of the output pairs, but by about $6^{\circ}$ in the third output pair

\section{STAR TRACKER CAMERA}

With the help of an NSF grant to upgrade system components at IOTA, we recently installed a new star-tracker CCD camera that was built to our specifications at MIT Lincoln Laboratories. The new system, called LLiST, is described in this volume by Schuller et al. ${ }^{26}$

Our goals in replacing our previous camera with LLiST were to obtain increased quantum efficiency, faster read-out speed, and lower read noise. The advantages we expect to gain are a greater dynamic range in star brightness and read-out speed, both of which are important for the large range of stars and conditions at IOTA. Preliminary tests suggest that we have achieved these goals in part, but that further fine-tuning will be needed to fully achieve our original intentions.

On quantum efficiency, we are sure that we now have at least a factor of two increase, as measured on stars; the previous system was a front-illuminated chip, and the current one is a back-side illuminated one, which explains the gain.

On read-out speed, the new system can be read out in binned mode about 5 times faster than the old system (about 1000 frames/sec vs 200). The CCD electronics will permit speeds up to 2000 frames/sec, but our software will need to be modified to handle that speed.

On read noise, the new system has a lower read noise in the unbinned (single-pixel) readout mode than the old sytem (about 7.5 electrons vs 10.1), but in binned mode (4x4 pixels) the noise is higher (about 16.5 electrons vs 10.1), which can likely be improved by some fine-tuning of the electronics.

An unexpected factor that we found is that the new chip (with a simple fan on the thermo-electric heat sink) does not get as cold as the previous chip (which had circulating chilled water on the heat sink). Therefore we plan to upgrade the new system with our old chilled water circulator, and we expect to drive down the dark current to the level where its noise is below the read noise.

\section{FACILITY EXPANSION}

In fall 2003 the Smithsonian Institution funded an expansion of the IOTA facility, and the project was completed in early 2004. The expansion provides us with a large control room that is isolated from the laboratory area, thus permitting separate heating and cooling controls, providing improved vibration isolation, and improved light-leakage control. The result is that we expect the beam-combining area to be more stable, with a gain in performance of the overall system.

\section{AUTO-ALIGNMENT}

In summer 2003 we installed a camera, optics, and light sources that are intended to allow us to automatically or semi-automatically carry out the daily fine-tuning of the optical system alignment, prior to each evening's observations. In the past we have done this by eye, with an alignment telescope. But the procedure sometimes requires an experienced eye, and new observers occasionally find themselves less than well equipped to recognize some of the visual clues that an experienced observer has learned. Therefore we decided to install flip-in LED guide lights at various points along the optical path, well centered on the intended optical axis, and to view these sequentially with a camera and frame-grabber. Corrections to the appropriate mirror at each point are made via commands to Pico-motors. At present we have installed most of the equipment needed to carry out this project; the remaining work is to complete the installation and teach the computer how to control the Pico-motors in response to the images obtained by the camera. 


\section{ACKNOWLEDGMENTS}

We thank the Smithsonian Institution for a Predoctoral Fellowship award to E. Pedretti, and NASA JPL for Michelson Postdoctoral Fellowships awarded to S. Ragland and E. Pedretti.

We thank NASA for its previous support of the 3-telescope upgrade project at IOTA, and the NSF for its sponsorship of our star-tracker upgrade project under grant number AST-0138303.

We thank Dr. Bernard B. Kosicki of the Advanced Imaging Technology Group at MIT Lincoln Laboratory for his help in this project.

Finally we thank the Smithsonian Institution for providing us with an upgraded facility at IOTA, as well as long-term operational support.

This publication made use of NASA's Astrophysics Data System (ADS) at http://adsabs.harvard.edu/.

\section{REFERENCES}

1. W. A. Traub, A. Ahearn, N. P. Carleton, J. Berger, M. K. Brewer, K. Hofmann, P. Y. Kern, M. G. Lacasse, F. Malbet, R. Millan-Gabet, J. D. Monnier, K. Ohnaka, E. Pedretti, S. Ragland, F. P. Schloerb, K. Souccar, and G. Weigelt, "New Beam-Combination Techniques at IOTA," in Interferometry for Optical Astronomy II. Wesley A. Traub, Ed., Proceedings of the SPIE, pp. 45-52, Feb. 2003.

2. E. Wilson, E. Pedretti, J. D. Bregman, R. Mah, and W. A. Traub, "Adaptive DFT-based fringe tracking and prediction at IOTA," in Proc. SPIE, New Frontiers in Stellar Interferometry, Wesley A. Traub; Ed., 5491, June 2004.

3. S. Kraus and F. P. Schloerb, "Infrared imaging of Capella with the IOTA interferometer," in Proc. SPIE, New Frontiers in Stellar Interferometry, Wesley A. Traub; Ed., 5491, June 2004.

4. E. Pedretti, N. D. Thureau, E. Wilson, W. A. Traub, J. Monnier, R. Millan-Gabet, and S. Ragland, "Fringe tracking at the IOTA interferometer," in Proc. SPIE, New Frontiers in Stellar Interferometry, Wesley A. Traub; Ed., 5491, June 2004.

5. S. Ragland et al., "Characterizing closure-phase measurements at IOTA," in Proc. SPIE, New Frontiers in Stellar Interferometry, Wesley A. Traub; Ed., 5491, June 2004.

6. J. Berger et al., "New IOTA observations of the pre-main sequence star FU Orionis with IONIC-3," in Proc. SPIE, New Frontiers in Stellar Interferometry, Wesley A. Traub; Ed., 5491, June 2004.

7. W. A. Traub, N. P. Carleton, J. D. Bregman, M. K. Brewer, M. G. Lacasse, P. Maymounkov, R. MillanGabet, J. D. Monnier, S. Morel, C. D. Papaliolios, M. R. Pearlman, I. L. Porro, F. P. Schloerb, and K. Souccar, "Third telescope project at the IOTA interferometer," in Proc. SPIE, Interferometry in Optical Astronomy, Pierre J. Lena; Andreas Quirrenbach; Eds., 4006, pp. 715-722, July 2000.

8. J. P. Berger, P. Haguenauer, P. Kern, K. Perraut, F. Malbet, I. Schanen, M. Severi, R. Millan-Gabet, and W. Traub, "Integrated optics for astronomical interferometry. IV. First measurements of stars," A\&A 376, pp. L31-L34, Sept. 2001.

9. J. D. Monnier, J. P. Berger, R. Millan-Gabet, W. A. Traub, N. P. Carleton, E. Pedretti, C. M. Coldwell, and C. D. Papaliolios, "SMART Precision Interferometry at $794 \mathrm{~nm}$," in Proc. SPIE, Interferometry for Optical Astronomy, Wesley A. Traub, editor, 4838, pp. 1127-1138, Aug. 2003.

10. E. Pedretti, R. Millan-Gabet, J. D. Monnier, W. A. Traub, N. P. Carleton, J.-P. Berger, M. G. Lacasse, F. P. Schloerb, and M. K. Brewer, "The PICNIC Interferometry Camera at IOTA," PASP 116, pp. 377-389, Apr. 2004.

11. P. Kervella, V. Coudé du Foresto, G. Perrin, M. Schöller, W. A. Traub, and M. G. Lacasse, "The angular diameter and distance of the Cepheid $\zeta$ Geminorum," A\&A 367, pp. 876-883, Mar. 2001.

12. B. Mennesson, G. Perrin, G. Chagnon, V. C. d. Foresto, S. Ridgway, A. Merand, P. Salome, P. Borde, W. Cotton, S. Morel, P. Kervella, W. Traub, and M. Lacasse, "Evidence for Very Extended Gaseous Layers around O-rich Mira Variables and M Giants," ApJ 579, pp. 446-454, Nov. 2002.

13. G. Chagnon, B. Mennesson, G. Perrin, V. Coudé du Foresto, P. Salomé, P. Bordé, M. Lacasse, and W. Traub, "L'-Band Interferometric Observations of Evolved Stars," AJ 124, pp. 2821-2832, Nov. 2002. 
14. K.-H. Hofmann, U. Beckmann, T. Blöcker, V. Coudé du Foresto, M. Lacasse, B. Mennesson, R. MillanGabet, S. Morel, G. Perrin, B. Pras, C. Ruilier, D. Schertl, M. Schöller, M. Scholz, V. Shenavrin, W. Traub, G. Weigelt, M. Wittkowski, and B. Yudin, "Observations of Mira stars with the IOTA/FLUOR interferometer and comparison with Mira star models," New Astronomy 7, pp. 9-20, Jan. 2002.

15. J. D. Monnier, R. Millan-Gabet, P. G. Tuthill, W. A. Traub, N. P. Carleton, V. Coude du Foresto, W. C. Danchi, M. G. Lacasse, S. Morel, G. Perrin, and I. Porro, "Aperture synthesis using multiple facilities: Keck aperture masking and the IOTA interferometer," in Proc. SPIE, Interferometry for Optical Astronomy, Wesley A. Traub, editor, 4838, pp. 379-386, Aug. 2003.

16. K. Ohnaka, U. Beckman, J. P. Berger, M. K. Brewer, K. Hofmann, M. G. Lacasse, V. Malanushenko, R. Millan-Gabet, E. Pedretti, J. D. Monnier, D. Schertl, F. P. Schloerb, V. Shenavrin, and W. A. Traub, "IOTA observation of the circumstellar envelope of $\mathrm{R} \mathrm{CrB}$," in Proc. SPIE, Interferometry for Optical Astronomy, Wesley A. Traub, editor, 4838, pp. 1068-1071, Aug. 2003.

17. R. Millan-Gabet, E. Pedretti, J. D. Monnier, W. A. Traub, F. P. Schloerb, N. P. Carleton, S. Ragland, M. G. Lacasse, W. C. Danchi, P. Tuthill, G. Perrin, and V. Coudé du Foresto, "Recent Science Results from the Two-Telescope IOTA," in Proc. SPIE, Interferometry for Optical Astronomy, Wesley A. Traub, editor, 4838, pp. 202-209, Aug. 2003.

18. G. Weigelt, U. Beckman, J. P. Berger, T. Bloecker, M. K. Brewer, K. Hofmann, M. G. Lacasse, V. Malanushenko, R. Millan-Gabet, J. D. Monnier, K. Ohnaka, E. Pedretti, D. Schertl, F. p. Schloerb, M. Scholz, W. A. Traub, and B. Yudin, "JHK-band spectro-photometry of T Cep with the IOTA interferometer," in Proc. SPIE, Interferometry for Optical Astronomy, Wesley A. Traub, editor, 4838, pp. 181-184, Aug. 2003.

19. J. D. Monnier, W. A. Traub, F. P. Schloerb, R. Millan-Gabet, J.-P. Berger, E. Pedretti, N. P. Carleton, S. Kraus, M. G. Lacasse, M. Brewer, S. Ragland, A. Ahearn, C. Coldwell, P. Haguenauer, P. Kern, P. Labeye, L. Lagny, F. Malbet, D. Malin, P. Maymounkov, S. Morel, C. Papaliolios, K. Perraut, M. Pearlman, I. L. Porro, I. Schanen, K. Souccar, G. Torres, and G. Wallace, "First Results with the IOTA3 Imaging Interferometer: The Spectroscopic Binaries $\lambda$ Virginis and WR 140," ApJ 602, pp. L57-L60, Feb. 2004.

20. P. Schuller, P. Salomé, G. Perrin, B. Mennesson, G. Niccolini, P. de Laverny, S. T. Ridgway, V. Coudé du Foresto, and W. A. Traub, "Are dust shell models well-suited to explain interferometric data of late-type stars in the near-infrared?," A\&A 418, pp. 151-162, Apr. 2004.

21. J. Berger, P. Haguenauer, P. Y. Kern, K. Rousselet-Perraut, F. Malbet, S. Gluck, L. Lagny, I. SchanenDuport, E. Laurent, A. Delboulbe, E. Tatulli, W. A. Traub, N. Carleton, R. Millan-Gabet, J. D. Monnier, E. Pedretti, and S. Ragland, "An integrated-optics 3-way beam combiner for IOTA," in Interferometry for Optical Astronomy II. Wesley A. Traub, Ed., Proceedings of the SPIE, 4838, pp. 1099-1106, Feb. 2003.

22. S. Ragland et al., "First Surface-resolved Results with the IOTA Imaging Interferometer: Detection of Asymmetric Structures in AGB Stars," ApJ , 2004. submitted.

23. W. D. Cotton, B. Mennesson, P. J. Diamond, G. Perrin, V. Coudé du Foresto, G. Chagnon, H. J. van Langevelde, S. Ridgway, R. Waters, W. Vlemmings, S. Morel, W. Traub, N. Carleton, and M. Lacasse, "VLBA observations of SiO masers towards Mira variable stars," A\&A 414, pp. 275-288, Jan. 2004.

24. D. D. S. Hale, W. Fitelson, J. D. Monnier, J. Weiner, and C. H. Townes, "Techniques for measuring phase closure at 11 microns," in Interferometry for Optical Astronomy II. Edited by Wesley A. Traub. Proceedings of the SPIE., 4838, pp. 387-397, Feb. 2003.

25. G. Perrin et al., "Interferometric Observations of Mira Stars in Narrow Bands in the Near-Infrared," A\&A , 2004. accepted.

26. P. Schuller, M. G. Lacasse, D. Lydon, E. Pedretti, F. P. Schloerb, and W. A. Traub, "LLiST - a new star tracker camera for tip-tilt correction at IOTA," in Proc. SPIE, New Frontiers in Stellar Interferometry, Wesley A. Traub; Ed., 5491, June 2004. 